\title{
A localization index for distinction between extracellular and intracellular antigens of Mycobacterium tuberculosis
}

\author{
Harald G. Wiker, ${ }^{1 *}$ Morten HarboE ${ }^{1}$ and Sadamu NAGaI ${ }^{2}$ \\ ${ }^{1}$ Institute of Immunology and Rheumatology, University of Oslo, Fr. Qvamsgt. 1, 0172 Oslo 1, Norway \\ ${ }^{2}$ Toneyama Institute for Tuberculosis Research, Osaka City University Medical School, Toneyama 5-1-1, Toyonaka, \\ Osaka 560, Japan
}

(Received 9 November 1990; accepted 27 November 1990)

\begin{abstract}
A series of mycobacterial antigens were quantified by immunoelectrophoresis, enzyme-linked immunosorbent assays, or SDS-PAGE with immunoblotting using antisera against purified mycobacterial antigens. The antigens showed a characteristic distribution profile. Some had a marked quantitative dominance in the culture fluid while others had a marked dominance in sonicates of whole washed bacilli. The majority of the antigens tested could thus be located and grouped as either secreted or cytoplasmic in terms of a localization index (LI) which is described. A 5-week-old Mycobacterium tuberculosis culture fluid preparation with a low degree of lysis was valuable in the delineation of localization indexes. The various secreted antigens showed a great span in LI values, from 5 to 1000 . This variation may express different degrees of secretion efficiency or differences in tendency to adhere to the bacterial surface. The identification of proteins as extracellular or cytosolic according to their LI values was in agreement for cultures of $M$. tuberculosis with a low degree of lysis and cultures of $M$. bovis BCG and $M$. bovis AN-5 with significant lysis of the bacterial cells.
\end{abstract}

\section{Introduction}

Proteins and other components of bacteria are differently localized in the bacterial cell. Some components are soluble constituents of the cytosol, some are bound to the cytoplasmic membrane, while others are associated with the cell wall or the capsule. Still other proteins are actively secreted and released, and are only transiently present in cell compartments.

Antigen localization is crucial in diseases in which antibodies are known to be essential mediators of the protective host response. Antibodies inactivate exotoxins or mediate lysis or phagocytosis of the infecting organism by binding to surface antigens. In diseases in which cell-mediated immunity plays an essential role, as in mycobacterial disease, very little is known about the nature of the protective antigens and whether their localization in the micro-organism is important.

Living mycobacteria are significantly more effective as inducers of protective immunity than dead bacilli (Bloch \& Segal, 1955). Various hypotheses have been put

\footnotetext{
Abbreviations: CIE, crossed immunoelectrophoresis; RIE, rocket immunoelectrophoresis; TEMED, $N, N, N^{\prime}, N^{\prime}$-tetramethylethylenediamine.
}

forward to explain this observation. An intriguing possibility is that viable mycobacteria release several antigens by active secretion and that secreted antigens are essential for induction of protective immunity. Some of these antigens have been identified (Closs et al., 1980; Fukui et al., 1965; Harboe \& Nagai, 1984; Nagai et al., 1981; Wiker et al., 1986a, $b, 1990 b$ ). For those which have been cloned, signal sequences responsible for their translocation have been revealed (see Table 1, which includes references).

The antigens $85 \mathrm{~A}$ and $85 \mathrm{~B}$ have been shown to bind human fibronectin (Abou-Zeid et al., 1988a). In $M$. tuberculosis, the antigen 85 complex is a major secreted constituent (Abou-Zeid et al., 1988b; Wiker et al., 1988). In $M$. bovis culture fluid, the most prominent antigen is MPB70 (Abou-Zeid et al., 1986; Harboe \& Nagai, 1984; Nagai et al., 1981).

Other proteins have also been cloned and sequenced, including the heat-shock proteins of $12 \mathrm{kDa}$ (MPT57) and $65 \mathrm{kDa}$ (antigen 82), which are GroES and GroEL homologues (Baird et al., 1988; Shinnick et al., 1988; Yamaguchi et al., 1988). It is important to note that their genes do not code for leader peptides involved in the secretion process. Detailed molecular studies of the 
$65 \mathrm{kDa}$ antigen showed that it is a soluble antigen with no extended hydrophobic regions, making it unlikely that it is an integral membrane protein (Shinnick et al., 1988). Therefore these proteins are most likely to be intracellular and in this study we used antigen 82 and MPT57 (or its homologue in M. bovis, MPB57) as markers for intracellular proteins.

The aim of this study was to introduce an index expressing the relative concentration of mycobacterial antigens located extra- and intracellularly and to locate individual mycobacterial antigens by way of this index. For this purpose we employed widely used culture fluid and sonicate preparations. We also showed that it is not necessary to use cultures where no bacterial lysis has occurred in order to be able to identify secreted antigens.

\section{Methods}

Bacteria. Mycobacterium bovis BCG Copenhagen (strain 1331), $M$. tuberculosis H37Rv (ATCC 27294) and M. bovis AN-5 were grown in the wholly synthetic Sauton medium for 3, 5 and 9 weeks, respectively. The bacilli were removed by centrifugation and the culture supernatant concentrated by $80 \%$ saturation ammonium sulphate precipitation. The bacilli were washed three times in phosphate-buffered saline
(PBS), and then sonicated in a rosette cooling cell with a Branson sonifier model B12 as described previously (Closs et al., 1980). Triplicate $200 \mathrm{ml}$ cultures of BCG Copenhagen were harvested at alternating 3 and $4 \mathrm{~d}$ intervals until day 25 to study bacillary growth and appearance of secreted and intracellular antigens in the culture fluid.

Antigens. The MPT antigens were purified by combining gel filtration and ion-exchange methods as described previously (Nagai $e t$ al., 1991). The purified recombinant $65 \mathrm{kDa}$ protein (Thole et al., 1985) was kindly supplied by Jan van Embden (Bilthoven, The Netherlands). The $65 \mathrm{kDa}$ antigen is termed antigen 82 throughout the paper because it has been identified as this precipitate line in the BCG reference system in crossed immunoelectrophoresis (De Bruyn et al., 1987a; Harboe et al., 1985). Protein concentrations were determined by measuring absorbance at $280 \mathrm{~nm}$ assuming $A_{\mathrm{cm}}^{1 \%}=10$, or by the Lowry method.

Antibodies. Polyclonal, monospecific rabbit antibodies were obtained either by immunizing with single precipitates obtained by crossed immunoelectrophoresis (CIE) (Harboe et al., 1976) or with soluble antigen using our general immunization procedure (Harboe $e t$ al., 1977). Table 1 summarizes the antibodies used, with their specificities and source. All contributors of antibody reagents are gratefully acknowledged. Polyvalent rabbit anti-BCG was kindly provided by DAKO Immunoglobulins, Copenhagen, Denmark (lot number 063). Polyvalent rabbit anti- $M$. tuberculosis serum was obtained by immunizing rabbits repeatedly with $1 \mathrm{mg}$ protein of a $M$. tuberculosis culture fluid antigen with a low content of intracellular antigens.

Table 1. Antibodies and mycobacterial antigens studied

\begin{tabular}{|c|c|c|c|c|c|c|}
\hline $\begin{array}{c}\text { Antigen/alternative } \\
\text { designation }{ }^{\dagger} \dagger\end{array}$ & $\begin{array}{l}\text { Mol. } \\
\text { mass } \\
(\mathrm{kDa})\end{array}$ & $\mathrm{LI}$ & Method $\ddagger$ & Secreted & $\begin{array}{c}\text { Signal } \\
\text { sequence } \dagger\end{array}$ & Antibodies* + \\
\hline MPT576/BCGa (11) & 12 & $<0.04$ & $\mathbf{R}$ & No & No $(12,13)$ & $\mathrm{K} 63^{8} \mathrm{RPab}$ anti-MPT57 \\
\hline MPT $46^{6}$ & 14 & 1 & $\mathbf{R}$ & $?$ & & K60 ${ }^{8}$ RPab anti-MPT46 \\
\hline MPT53 $^{6}$ & 15 & $>70$ & $\mathbf{R}$ & Yes & & $\mathrm{K} 62^{8}$ RPab anti-MPT53 \\
\hline MPT63 $^{6}$ & 18 & $>150$ & $\mathbf{R}$ & Yes & & K64 ${ }^{8}$ Rab anti-MPT63 \\
\hline MPB70 & 23 & 1000 & $\mathbf{R}$ & Yes & Yes (14) & K216 ${ }^{8}$ RPab anti-MPB70 (15) \\
\hline MPT64 $^{6}$ & 26 & 40 & $\mathbf{R}$ & Yes & Yes (16) & $\mathrm{K} 97^{8} \mathrm{RPab}$ anti-MPB64 (17) \\
\hline MPT51 $^{\circ}$ & 27 & 25 & $\mathbf{R}$ & Yes & & K61 ${ }^{8}$ RPab anti-MPT51 \\
\hline $\begin{array}{l}\text { Antigen } 85 \mathrm{~B}^{8} / \mathrm{MPT}^{2} 9^{6} / \\
\text { alpha antigen }(20)\end{array}$ & 30 & 70 & R & Yes & Yes (18) & $\begin{array}{l}\text { K } 1031 \text { B }^{8} \text { RPab anti-MPT59 (19); } \\
\text { HYT2 } 27^{1} \text { Mab (21) }\end{array}$ \\
\hline $85 \mathrm{~A}^{8} / \mathrm{MPT}^{6} 4^{6} / \mathrm{P}^{32}(23)$ & 31 & 150 & $\mathbf{R}$ & Yes & Yes (24) & $\mathrm{K} 19^{8} \mathrm{RPab}$ anti-BCG85A \\
\hline Antigen $85 \mathrm{C}^{8} / \mathrm{MPT}^{4} 5^{6}$ & 31.5 & 20 & $\mathbf{R}$ & Yes & & $\mathrm{K} 20^{8} \mathrm{RPab}$ anti-BCG85C \\
\hline Antigen 78/antigen $5^{3}(25)$ & 38 & 5 & $\mathbf{R}$ & Yes & Yes (26) & $\mathrm{Q} 1^{3}$ GPab anti-antigen 5 \\
\hline Antigen $84^{7}$ & 36 & $<0 \cdot 1$ & $\mathrm{E}$ & No & & No. $10^{7} \mathrm{RPab}$ anti-34kD antigen $\mathrm{F}-126-2^{5} \mathrm{Mab}$ \\
\hline MPT $32^{6}$ & 42 & 90 & $\mathbf{R}$ & Yes & & K598 RPab anti-MPT32 \\
\hline Antigen $10^{8}$ & 43 & 0.04 & $\mathbf{R}$ & No & & K $1029 B^{8}$ RPab anti-antigen 10 \\
\hline Antigen $82 / 65$ kD $/$ P64 (27) & 65 & 0.0004 & $\mathrm{E}$ & No & No $(28)$ & $\mathrm{K} 57^{8} \mathrm{RPab}$ anti-antigen $82 \mathrm{RPab}, \mathrm{TB} 78^{2} \mathrm{Mab}$ \\
\hline Antigen $63 / \mathrm{hsp} 70(29)$ & 70 & $<0.03$ & $\mathrm{C}$ & No & & \\
\hline
\end{tabular}

* Sources of antigens and antisera: 1, J. Bennedsen (Copenhagen, Denmark); 2, A. R. M. Coates (London, UK); 3, T. M. Daniel (Cleveland, Ohio, USA); 4, J. van Embden (Bilthoven, The Netherlands); 5, A. H. J. Kolk (Amsterdam, The Netherlands); 6, S. Nagai; 7, M. E. Patarroyo (Bogota, Colombia); 8, H. G. Wiker/M. Harboe.

$\dagger$ References: 11, Minden et al. (1984); 12, Baird et al. (1988); 13, Yamaguchi et al. (1988); 14, Terasaka et al. (1989); 15, Harboe \& Nagai (1984); 16, Yamaguchi et al. (1989); 17, Harboe et al. (1986); 18, Matsuo et al. (1988); 19, Wiker et al. (1990a); 20, Tasaka et al. (1983); 21, Schou et al. (1985); 22, Wiker et al. (1986a); 23, De Bruyn et al. (1987b); 24, Borremans et al. (1989); 25, Daniel \& Anderson (1978); 26, Andersen \& Hansen (1989); 27, De Bruyn et al. (1987a); 28, Shinnick et al. (1988); 29, Garsia et al. (1989).

$\ddagger$ E, enzyme linked immunosorbent assay; $R$, rocket immunoelectrophoresis; $C$, crossed immunoelectrophoresis.

Abbreviations: R Pab, rabbit polyclonal antibodies; Mab, mouse monoclonal antibodies; GPab, goat polyclonal antibodies; MPT, $M$. tuberculosis protein (the value defines the relative electrophoretic mobility at $\mathrm{pH} 9.5$ in $7.7 \%$ polyacrylamide gel: Nagai et al., 1981). 
Enzyme-linked immunosorbent assay (ELISA). A double sandwich antibody ELISA for quantification of antigen 85 has been described in detail previously (Wiker et al., 1990a). Briefly, Nunc-Immunoplate II (lot 0984) wells were coated with the mouse monoclonal IgGI antibody, HYT27. Triplicates of different dilutions of antigen were applied and then rabbit antiserum against antigen 85 . In the last step, donkey antirabbit immunoglobulin conjugated with horseradish peroxidase (Amersham) was applied. 2,2-Azino-di-(3-ethylbenzthiazolinsulphonate) (ABTS) was used as substrate, and the $A_{405}$ was read on a Dynatech MR580 plate reader. All incubations after the coating step were performed in PBS with $0.2 \%$ Tween 20 , and the plates were washed three times in a Dynatech Dynawasher between each incubation, with PBS containing $0.1 \%$ Tween 20 . A similar assay was developed for antigen 82 using the mouse monoclonal antibody TB78 ( $1: 1000$ dilution) as coating antibody and rabbit anti-antigen 82 serum $(1: 1000)$ after the antigen step. Dilutions of highly purified antigen 85B and antigen 82 at defined protein concentrations were used as standards.

Quantitative immunoelectrophoresis. Individual mycobacterial antigens were quantified by rocket immunoelectrophoresis (RIE) (Axelsen \& Bock, 1983) on $9 \times 11 \mathrm{~cm}$ glass plates with different monospecific rabbit antisera $(0.5-2 \mathrm{ml}$, depending on the antibody content) incorporated into $15 \mathrm{ml} \mathrm{1 \%}(\mathrm{w} / \mathrm{v})$ agarose (Litex-HSA, batch 3271$)$. Triton X-100 at a final concentration of $0.1 \%$ in the agarose was used for some antigens in the $M$. tuberculosis preparations. Twofold dilutions of culture fluid and sonicate preparations were tested on the same plate to determine the relative quantity of each antigen located extra versus intracellularly. A standard curve, plotted on semilogarithmic paper, was made from the rockets formed by the antigen preparation in which a particular antigen was most abundant (culture fluid or sonicate). A given rocket from the other antigen preparation was assigned the value of 1 and compared with the standard curve to determine the relative abundance in multiples of 1 . The values obtained were used for $x$ and $y$ or $m$ and $n$ in the equations I-IV for the localization index or index of lysis (see later in Methods).

CIE was performed on $5 \times 7 \mathrm{~cm}$ glass plates as described in detail previously (Closs et al., 1980). Immunoprecipitates were stained with Coomassie brilliant blue R-250.

Sodium dodecyl sulphate polyacrylamide gel electrophoresis (SDS$P A G E)$ with immunoblotting. SDS-PAGE (Laemmli, 1970) $(16 \times$ $18 \mathrm{~cm})$ was run with a $5 \%(\mathrm{w} / \mathrm{v})$ stacking gel and a $12.5 \%(\mathrm{w} / \mathrm{v})$ separating gel. BCG and $M$. tuberculosis culture fluids and sonicates were separated ( $40 \mu \mathrm{g}$ total protein in each lane) and blotted on to nitrocellulose. The nitrocellulose was blocked with $0 \cdot 2 \%$ Tween $20,1 \%$ $(w / v)$ gelatin and $2 \%(w / v)$ BSA in PBS, and washed between each incubation with $0.1 \%$ Tween 20 in PBS. Antigen bands were detected using different monospecific rabbit antisera against purified $M$. tuberculosis $\mathrm{H} 37 \mathrm{Rv}$ antigens diluted $1: 200$ or $1: 1000$. Acrylamide, bisacrylamide, TEMED, ammonium persulphate, and molecular mass markers were obtained from Bio-Rad. Bound rabbit antibodies were detected as with the ELISA. The substrate solution contained $20 \mathrm{mg} \mathrm{3-}$ amino-9-ethylcarbazol (Sigma) dissolved in $2.5 \mathrm{ml} \mathrm{N}, N$,-dimethylformamide (Merck) and $50 \mathrm{ml} 0.05 \mathrm{M}$-sodium acetate $\mathrm{pH} 5.0$ containing $0.015 \% \mathrm{H}_{2} \mathrm{O}_{2}$

Development of the mycobacterial antigen localization index $(L I)$. For localization of antigens we developed an index for individual antigens expressing the ratio between the amount of each antigen in culture fluid and in sonicate preparations.

$$
L I=\frac{\text { antigen as a fraction of total protein in the culture fluid }}{\text { antigen as a fraction of total protein in the sonicate }}
$$

If $x=$ concentration of the antigen in culture fluid (mg protein $\mathrm{ml}^{-1}$ ), $\mathrm{y}=$ concentration of the antigen in sonicate, $A=$ total protein concentration in culture fluid, and $B=$ total protein concentration in sonicate, the index becomes.

$$
\mathrm{LI}=\frac{x / A}{y / B}=\frac{\mathrm{B} \cdot \mathrm{x}}{\mathrm{A} \cdot \mathrm{y}}
$$

The index may be determined using this equation in an ideal situation with negligible lysis of bacteria during culture. If lysis has occurred, it is necessary to estimate the degree of bacterial lysis by assay of a 'marker' protein known to be a soluble intracellular antigen. The sonicate preparations, after washing of the bacilli, will not vary much with different degrees of lysis. In these instances, however, the culture fluid preparation will be contaminated with intracellular antigens.

If $B^{\prime}=$ the total protein concentration of intracellularly derived protein antigens appearing in the culture fluid, $A^{\prime}=$ the total protein concentration in the culture fluid of culture with bacterial lysis, $m=$ concentration of a soluble intracellular antigen in the culture fluid, and $n=$ concentration of the soluble intracellular antigen in the sonicate, the following equations may be used:

$$
A^{\prime}=A+B^{\prime} \text { and } B^{\prime}=\frac{m \cdot B}{n}
$$

which gives $A=A^{\prime}-\frac{m \cdot B}{n}$, and

$$
\mathrm{LI}=\frac{B \cdot x}{\left(A^{\prime}-\frac{m \cdot B}{n}\right) \cdot y}
$$

This second equation corrects for contamination with intracellular proteins in the culture fluid. However, the true value of $x$ must also be calculated. If $x^{\prime}=$ the measured concentration in the culture fluid of an antigen in a lysed culture, then

$$
x=x^{\prime}-\frac{m \cdot y}{n}
$$

$x$ may then be substituted for in equation II giving

$$
\mathrm{LI}=\frac{B \cdot\left(x^{\prime}-\frac{m \cdot y}{n}\right)}{\left(A^{\prime}-\frac{m \cdot B}{n}\right) \cdot y}
$$

It is important to include a control for the extent of lysis by concomitant assay of a soluble cytoplasmic antigen. Thus, a selected, exclusively cytoplasmic marker antigen is assayed to establish the values for $m$ and $n$ for any given culture studied. The index of lysis ' $I_{1 y s i s}$ ' may then be determined:

$$
\mathrm{I}_{\mathrm{lysis}}=\frac{B \cdot m}{A^{\prime} \cdot n}
$$

$I_{\mid y s i s}$ expresses the proportion of intracellular proteins in the culture fluid. Theoretically this index will always be lower than 1 . When $m=0$ the LI may be calculated directly from equation $I$.

In this study we used the intracellular MPT57/MPB57 and antigen 82 as cytoplasmic markers. It may be inadequate to use a single antigen to follow the behaviour of all intracellular proteins on cell lysis. Using these stress proteins may give an overestimate of lysis but this may be useful, a point discussed further in the Discussion.

Table 2 shows the effect of different degrees of lysis on different theoretical LI values calculated by equation III. It shows that a wrong estimation of the degree of lysis has only a modest effect on the resulting index value when lysis is small or moderate. As the lysis becomes greater the error becomes increasingly significant. 
Table 2. Effect on hypothetical LI values when calculating with different degrees of lysis

The influence of the degree of lysis on the hypothetical LI values indicated was calculated by using different $I^{\text {lysis }}$ values in equation III.

\begin{tabular}{llccccc}
\hline \hline & \multicolumn{5}{c}{ LI value } \\
\cline { 2 - 6 } $\begin{array}{c}\mathrm{I}_{\text {1,yis }} \\
\text { value } \\
\text { used }\end{array}$ & 0 & $\begin{array}{c}\text { Hypothetical } L I \\
0.5\end{array}$ & 1 & 2 & 10 \\
\hline 0.33 & -0.5 & 0.25 & 1 & $2 \cdot 5$ & 14 \\
0.50 & -1 & 0 & 1 & 3 & 19 \\
0.90 & -9 & -4 & 1 & 11 & 91 \\
\hline \hline
\end{tabular}

\section{Results}

\section{Appearance of antigens in culture filtrate}

BCG cultures were harvested at different ages; Fig. $1(a)$ shows growth of BCG on Sauton medium as indicated by wet weight of bacilli. Antigen 82, which we chose as a cytoplasmic marker, could not be detected at day 1,4 or 7 and was barely detectable at day 11 , but then its concentration increased rapidly towards the end of the exponential growth phase (Fig. 1b). Antigen 85B was readily detected at day 4 and its increase corresponded closely to the increase in bacterial mass (Fig. $1 b$ ). Another set of bacterial cultures gave the same overall result (data not shown). The gradual appearance of antigen 82 in the culture fluid demonstrates that the degree of lysis was related to the age of the culture.

\section{Comparison of culture fluid and sonicate preparations}

Culture fluid and sonicate antigens of BCG and $M$. tuberculosis were separated by SDS-PAGE and blotted on to nitrocellulose. The nitrocellulose sheets were then incubated with antisera to various mycobacterial proteins. Using monospecific rabbit antiserum against antigen 82 , multiple strong bands were seen in all preparations except the $M$. tuberculosis culture fluid, which was negative (Fig. $2 a$ ). The polyclonal antibodies used here reacted monospecifically with antigen 82 in CIE. However, antigen 82 is known to be easily degraded and multiple bands are frequently obtained with monoclonal antibodies as well (Young et al., 1987). In lane 2 strong bands are seen around $30 \mathrm{kDa}$. They represent major degradation products precipitated in the anodic peak of the double-peaked precipitate of antigen 82 in CIE (antigen 82B) (Wiker et al., 1988).

Fig. 2(b) shows immunoblotting with anti-antigen 85 serum. The pattern was completely different from that seen with anti-antigen 82 serum. With both culture fluid

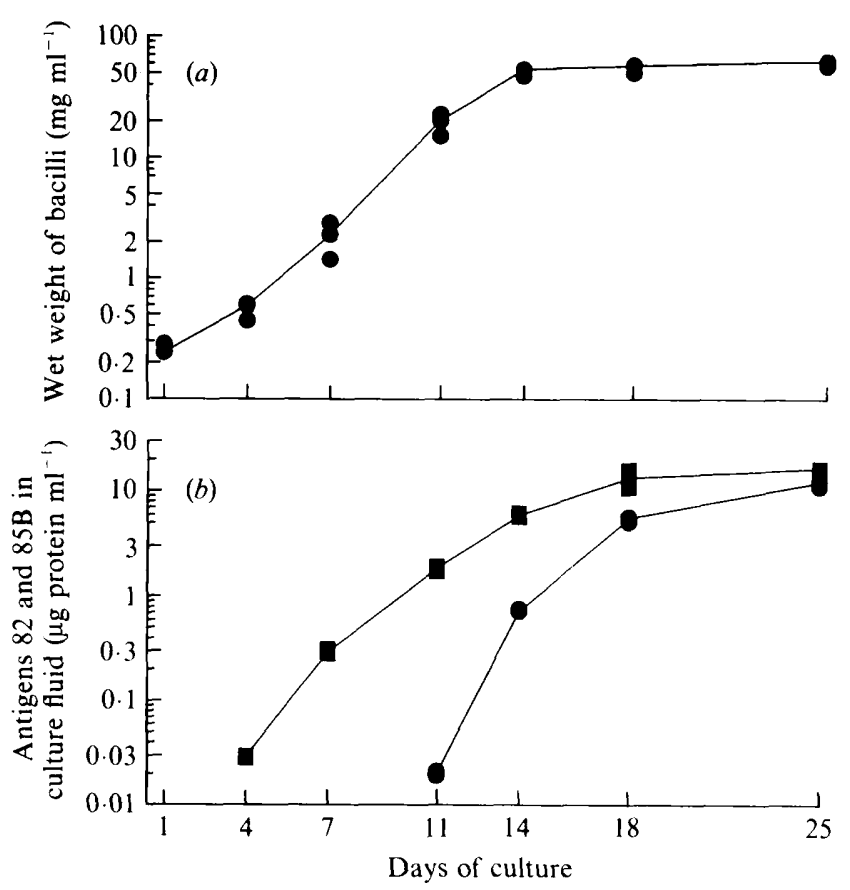

Fig. 1. Triplicate $200 \mathrm{ml}$ cultures of BCG Copenhagen on Sauton medium were harvested twice a week until day 25 . The bacilli were removed by filtration on Whatman paper no. 1 and weighed. The wet weight values are shown in $(a)$. After sterile filtration of the culture fluids, they were diluted $1: 100$ in PBS Tween and tested for concentration of antigen $82(\bullet)$ and antigen 85B $(\square)$ by ELISA $(b)$. For both $(a)$ and $(b)$, triplicate values are shown at each interval, but the symbols largely overlap due to close proximity of the values.

preparations there were doublets at 30 and $31 \mathrm{kDa}$ which appeared as single broad bands because of their strong reaction. The blot was developed to visualize the weak bands at 30 and $31 \mathrm{kDa}$ seen in both sonicate preparations.

Fig. 2(c) shows immunoblotting using an antiserum against MPT53. A prominent band at $15 \mathrm{kDa}$, which corresponds to the molecular mass of the isolated protein, was seen in both culture fluids, while only weak bands were seen in the sonicate preparations. Again the immunoblot was strongly developed, and a slight reactivity appeared against the antigen 85 complex at 30 and $31 \mathrm{kDa}$. Immunoblotting with the other antisera directed against secreted antigens all gave results with strong bands in the culture fluids and undetectable or weaker bands in the sonicates (data not shown). The results thus corresponded to the patterns seen with antiantigen 85 and anti-MPT53 sera.

Fig. 3 shows CIE of the $M$. tuberculosis culture fluid and its corresponding sonicate. The most prominent line in the culture fluid corresponds to antigen 85 (Fig. $3 a, b$ ). The anti-BCG serum gave fewer precipitate lines (Fig. $3 b$ ) than our new anti- $M$. tuberculosis culture fluid antiserum (Fig. 3a). Most of the lines seen in Fig. 3(a) 

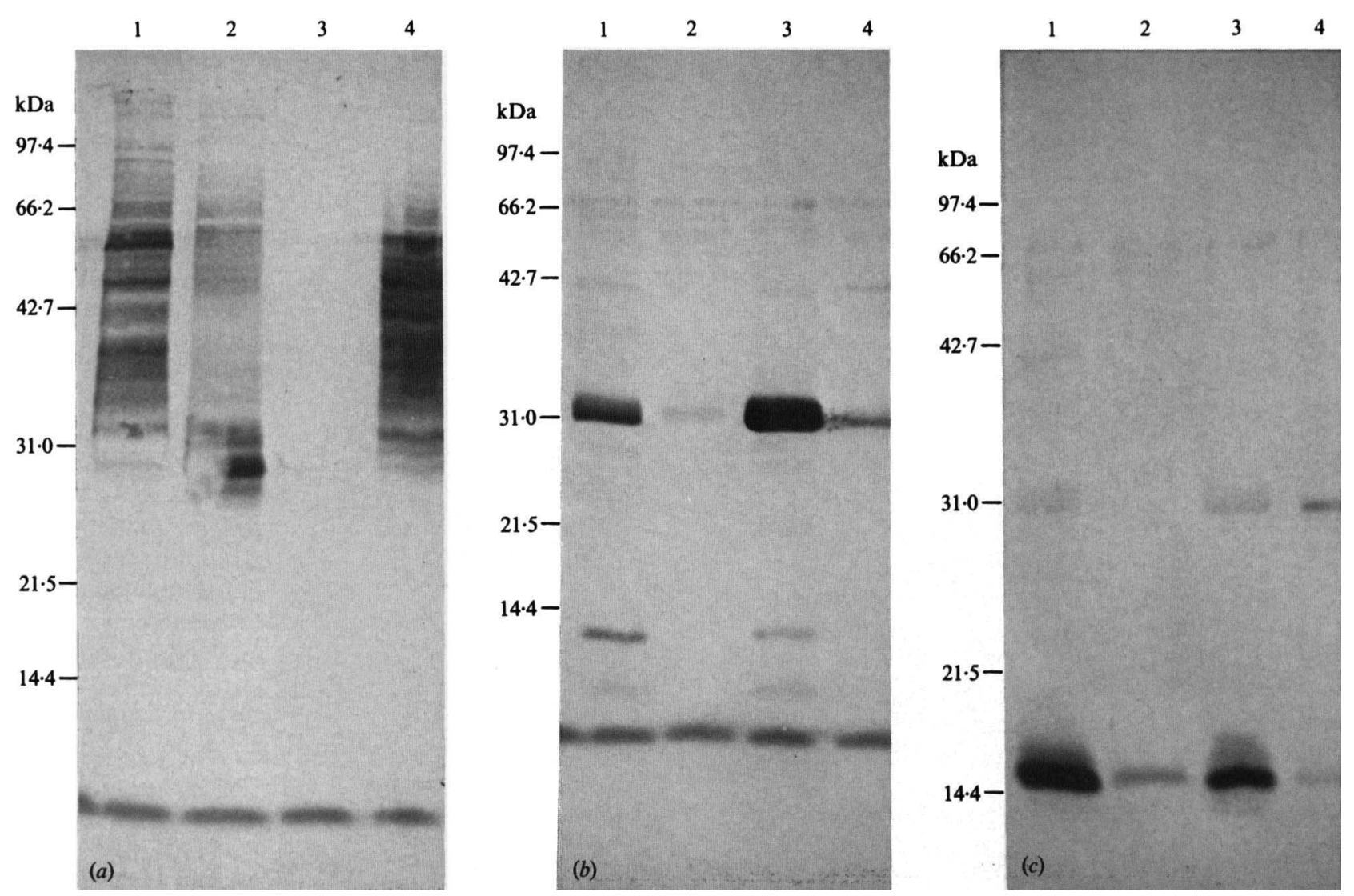

Fig. 2. Immunoblotting with specific rabbit antibodies against antigen $82(a)$, antigen $85(b)$ and MPT53 (c). Culture fluids and sonicates from BCG ( 3 weeks old) and $M$. tuberculosis ( 5 weeks old) were separated by SDS-PAGE and blotted on to nitrocellulose. Lanes 1 , BCG culture fluid; lanes 2, BCG sonicate; lanes $3, M$. tuberculosis culture fluid; lanes $4, M$. tuberculosis sonicate. The positions of the molecular mass markers are indicated.

correspond to secreted antigens; however, we cannot conclusively assign the lines with highest electrophoretic mobilities appearing in the intermediate gel. These antigens may reflect a low degree of lysis of the bacteria. The anti-BCG serum gave a complex pattern with the $M$. tuberculosis sonicate preparation (Fig. 3c). The relationship between the $M$. tuberculosis culture fluid and sonicate patterns was further investigated by incorporating large amounts of $M$. tuberculosis culture fluid into the intermediate gel (Fig. $3 d$ ). Several horizontal precipitates can be seen, some fusing with lines of the sonicate pattern. There are also several lines in the sonicate pattern which were not affected at all. Compared with Fig. $3(c)$ they appear in the same position, demonstrating that the $M$. tuberculosis culture fluid did not contain significant amounts of these antigens, and confirming that the majority of precipitate lines seen in the CIE with $M$. tuberculosis sonicate represent intracellular antigens.

The combined results obtained by ELISA, immunoblotting and CIE represent two different bacterial strains, and confirm that there is a strong distinction between extracellular and intracellular mycobacterial antigens. Two other culture fluid batches of the particular laboratory strain of $M$. tuberculosis were tested in CIE, confirming that this strain consistently provides cultures with a low degree of lysis. Comparison between the results obtained with BCG and $M$. tuberculosis also showed that the degree of lysis which occurred in mycobacterial cultures varied from strain to strain.

\section{Localization index and cell lysis}

Localization indices (LI) in $M$. tuberculosis for all antigens studied are summarized in Table 1. The data used for their calculation are shown separately for $M$. tuberculosis (Table 3), M. bovis BCG (Table 4) and $M$. bovis AN-5 (Table 5). $\mathrm{I}_{1 y s i s}$ values were calculated using MPT57/MPB57 or antigen 82 and were used in calculating LI values for $M$. bovis (Tables 4 and 5). For $M$. tuberculosis, five antigens which appeared cytosolic by their low LI values all gave low $\mathbf{I}_{\text {lysis }}$ values in 

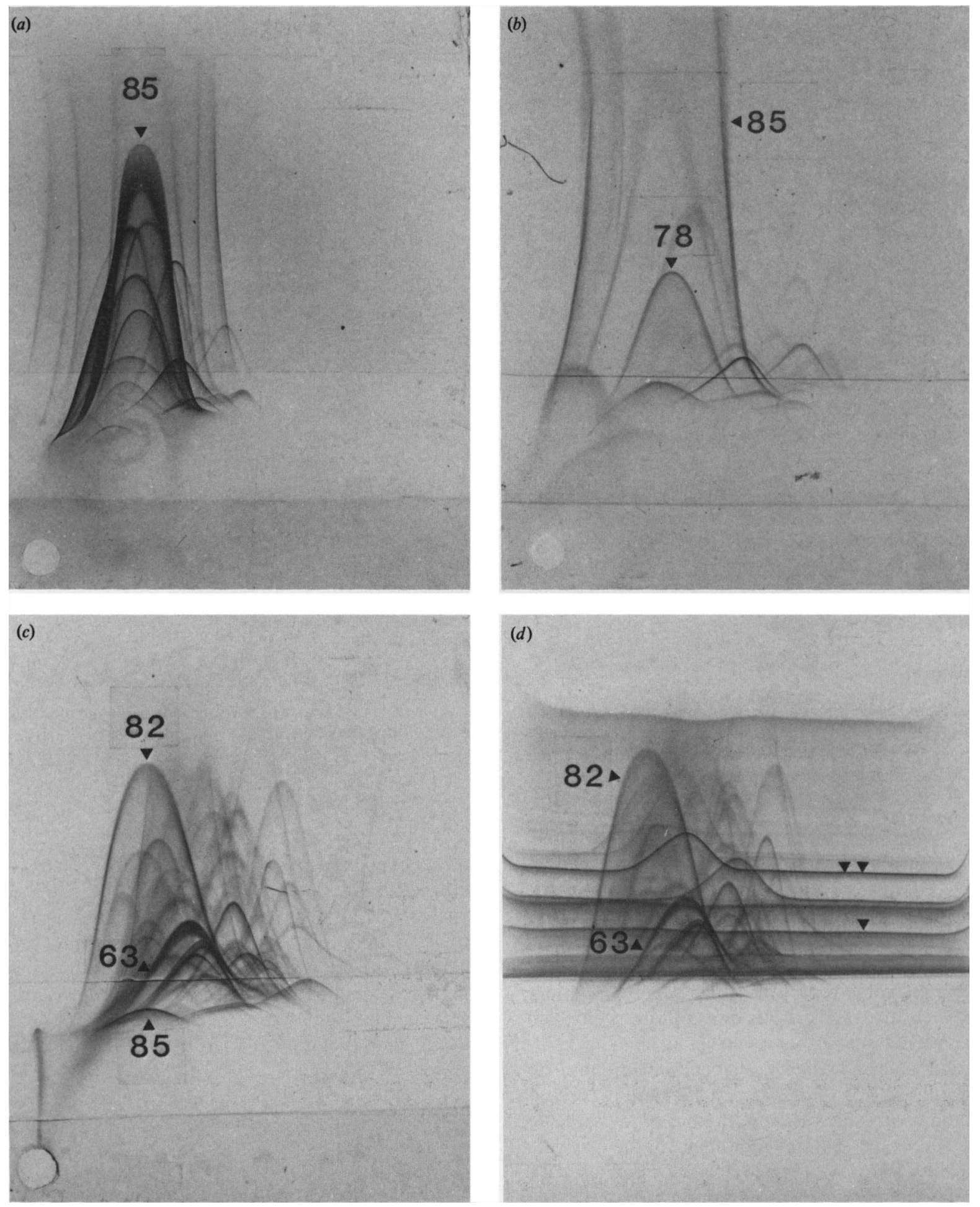

Fig. 3. CIE of $M$. tuberculosis antigen. The top gel contained (a) $200 \mu \mathrm{l}$ anti-M. tuberculosis culture fluid serum, or $(b, c, d) 200 \mu l$ antiBCG immunoglobulin. In $(a)$ and $(b)$ the antigen well (lower left corner) contained $30 \mu \mathrm{g} M$. tuberculosis culture fluid antigen; in (c) and (d) it contained $30 \mu \mathrm{g} M$. tuberculosis sonicate antigen. In (d) the intermediate gel contained $1.2 \mathrm{mg} M$. tuberculosis culture fluid antigen to reveal whether antigens of the $M$. tuberculosis sonicate pattern were present in the $M$. tuberculosis culture fluid. In $(c), 100 \mu 10 \cdot 1 \mathrm{M}$ $\mathrm{NaCl}$ was added to the intermediate gel as a control. The positions of relevant antigens are shown. In $(d)$, a single arrowhead marks an example of a horizontal precipitate line which did not fuse with any corresponding line in the sonicate pattern. Two arrowheads mark a horizontal precipitate line fusing with a precipitate line of the sonicate pattern, which has been markedly elevated from its original position in $(c)$. 
Table 3. LI values of defined antigens of $M$. tuberculosis in a 5-week-old culture as determined by quantitative immunoelectrophoresis

$A$ and $B$ give the total protein concentration $\left(\mathrm{mg} \mathrm{ml}^{-1}\right), m$ and $n$ (for cytoplasmic markers; equation IV), $x$ and $y$ (equation I) the individual antigen titres in culture fluid and sonicate preparations respectively (see Methods for further explanation). Antigens 82 and 84 were determined by double-antibody ELISA. $<1=$ antigen not detected in the antigen preparation. The index values are representative. The determinations were repeated for most of the secreted $M$. tuberculosis antigens, and the results used to calculate a standard deviation $( \pm 12.4 \%)$ to apply to all the index values.

\begin{tabular}{lccrrr}
\hline \hline Antigen* & $A^{\prime}$ & $B$ & $m$ & $n$ & $\mathrm{I}_{\text {lysis }}$ \\
\hline MPT 57 (1) & $12 \cdot 3$ & $7 \cdot 1$ & $<1$ & 16 & $<0 \cdot 04$ \\
Antigen 84 (1) & $12 \cdot 3$ & $7 \cdot 1$ & $<1$ & 6 & $<0 \cdot 1$ \\
Antigen 82 (1) & $12 \cdot 3$ & $7 \cdot 1$ & 1 & 1450 & $0 \cdot 0004$ \\
Antigen 63 (1) & $12 \cdot 3$ & $7 \cdot 1$ & $<1$ & 23 & $<0 \cdot 03$ \\
Antigen 10 (1) & $12 \cdot 3$ & $7 \cdot 1$ & 1 & 14 & $0 \cdot 04$ \\
& $A$ & $B$ & $x$ & $y$ & LI (eq. I) \\
MPT 46 (1) & $12 \cdot 3$ & $7 \cdot 1$ & 2 & 1 & 1 \\
Antigen 78 (1) & $11 \cdot 1$ & $6 \cdot 4$ & 8 & 1 & 5 \\
Antigen 85C (2) & $11 \cdot 1$ & $6 \cdot 4$ & 32 & 1 & 20 \\
MPT 51 (2) & $12 \cdot 3$ & $7 \cdot 1$ & 46 & 1 & 25 \\
MPT 64 (2) & $11 \cdot 1$ & $6 \cdot 4$ & 70 & 1 & 40 \\
Antigen 85B (3) & $11 \cdot 1$ & $6 \cdot 4$ & 128 & 1 & 70 \\
MPT 53 (3) & $12 \cdot 3$ & $7 \cdot 1$ & 128 & $<1$ & $>70$ \\
MPT 32 (2) & $12 \cdot 3$ & $7 \cdot 1$ & 160 & 1 & 90 \\
Antigen 85A (2) & $11 \cdot 1$ & $6 \cdot 4$ & 256 & 1 & 150 \\
MPT 63 (2) & $12 \cdot 3$ & $7 \cdot 1$ & 256 & $<1$ & $>150$ \\
\hline \hline
\end{tabular}

* Number of determinations in parentheses.

Table 4. LI values of defined antigens of $M$. bovis BCG in a 3-week-old culture as determined by quantitative immunoelectrophoresis

$A^{\prime}$ and $B$ give the total protein concentration $\left(\mathrm{mg} \mathrm{ml}^{-1}\right), m$ and $n$ (for cytoplasmic markers; equation IV), $x$ and $y$ (equation III) the individual antigen titres in culture fluid and sonicate preparations respectively. Other details as for Table 3 .

\begin{tabular}{lccccc}
\hline \hline Antigen* & $A^{\prime}$ & $B$ & $m$ & $n$ & $\mathrm{I}_{\text {ly sis }}$ \\
\hline MPB 57 (1) & 3 & 3 & 1 & $3 \cdot 1$ & $0 \cdot 32$ \\
Antigen 82 (1) & 3 & 3 & $2 \cdot 6$ & 8 & $0 \cdot 33$ \\
& & & & & LI \\
Antigen 85C (1) & $A^{\prime}$ & $B$ & $x$ & $y$ & (eq. III) \\
MPB 51 (1) & 3 & 3 & 14 & 1 & 20 \\
Antigen 85B (2) & 3 & 3 & 10 & 1 & 15 \\
MPB 53 (1) & 3 & 3 & 10 & 1 & 15 \\
MPB 32 (1) & 3 & 3 & 15 & $<1$ & $>95$ \\
Antigen 85A (1) & 3 & 3 & 28 & 1 & 20 \\
MPB 63 (1) & 3 & 3 & 64 & $<1$ & $>90$ \\
\hline \hline
\end{tabular}

* Number of determinations in parentheses.

agreement with each other. Most LI values are of secreted antigens since our rabbit antisera have been raised against antigens purified from culture fluids. Two antigens were not detected by RIE in any of the
Table 5. LI values of defined antigens of $M$. bovis $A N-5$ in a 9 week-old culture as determined by quantitative immunoelectrophoresis

$A^{\prime}$ and $B$ give the total protein concentration $\left(\mathrm{mg} \mathrm{ml}^{-1}\right), m$ and $n$ (for cytoplasmic markers; equation IV), $x$ and $y$ (equation III) the individual antigen titres in culture fluid and sonicate preparations respectively. The LI values were calculated by correcting for the extent of lysis using the $\mathrm{I}^{\text {lysis }}$ valure for antigen 82 . Other details as for Table 3 .

\begin{tabular}{llllcc}
\hline \hline Antigen* & $A^{\prime}$ & $B$ & $m$ & $n$ & $\mathrm{I}_{\mathrm{lysis}}$ \\
\hline Antigen 82 (1) & $7 \cdot 4$ & $4 \cdot 5$ & 1 & $3 \cdot 1$ & $0 \cdot 20$ \\
& & & & & LI \\
& $\boldsymbol{A}^{\prime}$ & $\boldsymbol{B}$ & $x$ & $y$ & (eq. III) \\
Antigen 85C (1) & $7 \cdot 4$ & $4 \cdot 5$ & 32 & $<1$ & $>20$ \\
MPB 51 (1) & $7 \cdot 4$ & $4 \cdot 5$ & 16 & 1 & 10 \\
MPB 64 (1) & $7 \cdot 4$ & $4 \cdot 5$ & 19 & 1 & 15 \\
Antigen 85B (1) & 3 & 3 & 16 & 1 & 20 \\
MPB 53 (1) & $7 \cdot 4$ & $4 \cdot 5$ & 32 & $<1$ & $>20$ \\
MPB 32 (1) & $7 \cdot 4$ & $4 \cdot 5$ & 16 & $<1$ & $>10$ \\
Antigen 85A (1) & $7 \cdot 4$ & $4 \cdot 5$ & 32 & $<1$ & $>20$ \\
MPB 63 (1) & $7 \cdot 4$ & $4 \cdot 5$ & 64 & $<1$ & $>45$ \\
MPB 70 (2) & 3 & $4 \cdot 5$ & 512 & 1 & 1000 \\
\hline \hline
\end{tabular}

* Number of determinations in parentheses.

tested sonicate preparations (MPT53/MPB53 and MPT63/MPB63). Antigens with a high LI value in $M$. tuberculosis also tended to have high LI values in BCG and $M$. bovis AN-5. Thus, the rank order of the LI values of individual proteins correlated when comparing the three strains despite different culture times and degrees of lysis. The standard deviation of the mean, $\pm 12.4 \%$, was somewhat higher than is usual with RIE; this is explained by the small quantities of secreted antigens found in the sonicates.

In the 9-week-old $M$. bovis AN-5 culture it was difficult to estimate the extent of lysis (Table 5). The MPB57 precipitates were weaker and the rockets higher in the culture fluid than in the sonicate. CIE of the culture fluid with anti-MPT57 antibodies in the top gel gave a diffuse precipitate of lower first-dimension electrophoretic mobility fusing with the strong precipitate at the 'correct' position. This pattern indicates that the MPB57 molecules are partly degraded in this culture fluid. Using intact MPB57 for estimation of $\mathrm{I}_{1 y \text { sis }}$ gave a lower value than by using antigen 82 which was employed to correct for the extent of lysis in $M$. bovis AN-5 in Table 5. It appears that old cultures should be avoided when estimating LI values because several antigens are substantially degraded. On the other hand, it appears that secreted antigens are signficantly more resistant to proteolytic degradation than intracellular antigens (cf. Fig. 2). This is in agreement with the situation in other bacterial species (Tai, 1986). In old cultures the consequence is that antigens with high LI values are more reliably determined than antigens with low LI 
values. MPB70 is known to be a stable protein which may even be precipitated from bovine PPD with antibodies prepared by immunization with the native molecule (Harboe et al., 1990), and MPB70 has the highest LI value so far observed (Table 5). This is consistent with the finding of Lounatmaa \& Brander (1990), who have shown by electron microscopy that MPB70 is located in the outermost layer of $M$. bovis BCG cell wall, possibly representing the paracrystalline surface layer protein. Interestingly, their observation accords with MPB70 being crystallizable even from partially purified protein preparations (Nagai et al., 1981).

\section{Discussion}

There were two major findings in the present investigation. Firstly, putative intracellular and extracellular antigens showed markedly different relative concentrations in the cytosol and culture fluid. Secondly, a wide range of index values was obtained for different secreted antigens.

It is often claimed that proteins occurring in culture fluids may be intracellular antigens appearing there as a result of bacterial lysis. This explanation may be excluded for candidate secreted antigens by the present indexing method. It is highly unlikely that an intracellular antigen will have an LI value of 10 or more.

Theoretically, it is important to note that correction for lysis by equation III does not classify antigens as 'intracellular' which appear as 'secreted' by equation I and vice versa. Table 2 shows clearly that LI values above or below 1 will stay as such after correction for lysis. The LI value of 1 is the absolute value which determines whether an antigen should be grouped as secreted or intracellular. In practice, this absolute value relies on correct estimation of total protein concentration of culture fluid and sonicate. Therefore, until more experience is obtained one should keep a safety margin, and consequently $L I$ values between $0 \cdot 2$ and 5 should be interpreted with caution even when handling cultures with a low degree of lysis.

In several instances the majority of antigens in the culture fluid appear to be soluble intracellular antigens. Most polyvalent anti-BCG and anti- $M$. tuberculosis immunoglobulins are directed towards intracellular antigens, with a few marked exceptions such as antigen 85 and antigen 78 (Fig. 3). Comparative analysis of sonicates and various culture fluid preparations of lysed M. tuberculosis and BCG by CIE (Closs et al., 1980; Wiker et al., 1988) demonstrated a large group of antigens appearing in culture fluids reflecting the antigen pattern in the corresponding sonicate. We have repeated the experiments with $\mathrm{BCG}$, and expressed in terms of the $I_{1 y s i s}$, several antigens in this group give approximately the same value under conditions of culture giving high yields of bacteria.

In stressed bacterial cells prone to lyse, heat-shock proteins are probably induced and increase in concentration in the cells prior to lysis. This will give an overestimate of the degree of lysis if one of them is used as indicator. For identification of secreted antigens this is an advantage because the risk of having false positives will be reduced. A disadvantage of the $65 \mathrm{kDa}$ heatshock protein, antigen 82 , as indicator is that it is more susceptible to degradation than other antigens (cf. the multiple bands in immunoblotting, Fig. 2a). This will tend to give an underestimation of the degree of lysis as was indicated from the $\mathrm{I}_{\text {lysis }}$ of antigen 82 in M. bovis AN5. Thus antigen 63 , which is also a heat-shock protein, may be a better indicator for estimating the extent of lysis. It is certainly difficult to give a correct estimate of lysis in old cultures and to obtain accurate LI values, and it is clear that the index will be more accurately determined using cultures with an $\mathrm{I}_{\text {lysis }}$ value of $<0.5$ (Table 2).

Erroneous estimation of total protein concentrations, varying efficacy of the washing procedure, and different age of the cultures may give rise to systematic errors without affecting the ordering of the antigens according to their measured LI values. This implies that $\mathrm{LI}$ values are most informative when several antigens can be compared in each batch of corresponding sonicate and culture fluid.

Our data support the conclusion that antigen 82 is an intracellular antigen in BCG and M. tuberculosis. AbouZeid et al. (1988b) showed that antigen 82 cannot be detected in short-term culture fluid preparations of $M$. tuberculosis harvested in the exponential growth phase. However, their detection of antigen 63 (monoclonal antibody L7) and MPT57 (monoclonal antibody SA-12) in these preparations may be related to the use of highly sensitive techniques.

De Bruyn et al. $(1987 a, 1989)$ found that P64 (the $65 \mathrm{kDa}$ heat-shock protein $=$ antigen 82 ) is released into the culture medium in significant quantities in zincdeficient cultures. Only minor quantities may be found in zinc-sufficient cultures. Since the corresponding gene has no signal sequence, the large amount of P64 in zincdeficient culture fluids is probably explained by gene induction under stress conditions combined with increased cell lysis. The amounts of P64 and P32 (antigen $85 \mathrm{~A}$ ) are given as $\mu \mathrm{g}$ per $\mathrm{mg}$ total culture fluid protein by DeBruyn et al. (1989). An interesting feature is that the concentration of P32 was consistently less in the zincdeficient compared to the zinc-sufficient cultures. This is in agreement with the prediction that contamination 
with intracellular antigens will tend to reduce the relative amount of the secreted antigens in culture fluid preparations.

As Table 1 shows, there was good agreement between occurrence of signal sequences and measured LI values. All secreted antigens except one had LI values higher than 10 ; antigen $78(38 \mathrm{kDa})$ had an LI value of 5 . Interestingly, this particular protein is probably lipid conjugated at its amino-terminal cysteine, favouring attachment to the bacterial surface (Young et al., 1990). Our findings support this view. The other secreted proteins studied here are probably of a different kind since they have significantly higher LI values than antigen 78 .

Several factors may contribute to the wide range of LI values obtained for different secreted antigens. There was no apparent correlation between LI values and the molecular mass of secreted proteins (Table 1). Antigens $85 \mathrm{~A}$ and $85 \mathrm{~B}$ differed significantly in their LI values. Since both these antigens occur in high concentration in culture fluids this difference is unlikely to be caused solely by differences in protein expression. Another possibility is that the secreted antigens may differ in their tendency to adhere to the bacterial surface. For antigen $85 \mathrm{~B}$, however, it was not possible to alter its $\mathrm{LI}$ value significantly using further extraction with $0 \cdot 2 \mathrm{M}-\mathrm{NaCl}$ or $1 \%$ Triton X-100, or by digesting the bacilli with proteases such as lysozyme, proteinase $\mathrm{K}$ or trypsin prior to sonication. This indicates that the variation in LI values between these proteins is mainly due to variation in secretion efficiency between individual proteins rather than to different tendencies to adhere to the bacterial surface. However, more extensive and refined experiments are needed to distinguish these alternatives.

It would be of great interest to study whether protective immune responses induced by mycobacterial antigens show some preference for antigens with high or low $\mathrm{LI}$ values. The low $\mathrm{LI}$ value of the secreted antigen 78 could make it a target antigen for protective immune responses, especially if this antigen is firmly located at the bacterial surface. On the other hand, secreted antigens with high LI values may be more readily presented by macrophages harbouring living bacilli than antigens with low LI values, while intracellular antigens might not be presented unless the bacilli die and release them by lysis. Whether this argument is relevant could be tested experimentally by comparing development of cellmediated immune responses to defined secreted and intracellular antigens after infection with live mycobacterial cells.

This study shows that there is a relatively large group of secreted mycobacterial antigens. High LI values and signal sequences so far clearly define 12 secreted antigens. Our new anti- $M$. tuberculosis culture fluid serum obtained by immunizing with $M$. tuberculosis culture fluid, with negligible bacterial lysis, contains antibodies reacting strongly with a variety of secreted antigens (Fig. $3 a$ ) and will be valuable in further identification and characterization of these antigens.

This work was supported by grants from the Anders Jahres Fund for the Promotion of Science, the Norwegian Research Council for Science and the Humanities, the Laurine Maarschalk Fund, the Osaka Foundation for Promotion of Clinical Immunology, and by the Immunology of Leprosy (IMMLEP) Component of the United Nations Development Programme/World Bank/World Health Organization Special Programme for Research and Training in Tropical Diseases through provision of recombinant $65 \mathrm{kDa}$ protein and monoclonal antibodies to mycobacterial proteins.

We wish to thank Ms Gunni Ulvund and Mr Reidar Bjørlo for technical assistance, Ms Kari Bertelsen and Ms Suzanne Garman-Vik for help with the manuscript, Dr Finn Saxegaard at the National Veterinary Institute, Oslo, Norway, for providing mycobacterial cultures, Dr Leif Schjerven at the Animal Department for immunization of rabbits, and the Phototechnical Laboratory for illustration work.

\section{References}

Abou-Zeid, C., Smith, I., Grange, J. M., Steele, J. \& Rook, G. A. W. (1986). Subdivision of daughter strains of Bacille Calmette-Guérin (BCG) according to secreted protein patterns. Journal of General Microbiology 132, 3047-3053.

Abou-Zeid, C., Ratliff, T. L., Wiker, H. G., Harboe, M., BENNEDSEN, J. \& Rook, G. A. W. (1988a). Characterization of fibronectin-binding antigens released by Mycobacterium tuberculosis and Mycobacterium bovis BCG. Infection and Immunity 56, 30463051 .

Abou-Zeid, C., Smith, I., Grange, J. M., Ratliff, T. L., Steele, J. \& RooK, G. A. W. (1988b). The secreted antigens of Mycobacterium tuberculosis and their relationship to those recognized by the available antibodies. Journal of General Microbiology 134, 531-538.

ANDERSEN, A. B. \& HANSEN, E. B. (1989). Structure and mapping of antigenic domains of protein antigen $\mathrm{b}$, a 38,000-molecular-weight protein of Mycobacterium tuberculosis. Infection and Immunity 57, 2481-2488.

AXELsen, N. H. \& BocK, E. (1983). Electroimmunoassay (rocket immunoelectrophoresis). Scandinavian Journal of Immunology (suppl. 10), 17, 103-106.

Baird, P. N., Hall, L. M. C. \& Coates, A. R. M. (1988). A major antigen from Mycobacterium tuberculosis which is homologous to the heat shock proteins groES from $E$. coli and the htp $A$ gene product of Coxiella burnetii. Nucleic Acids Research 16, 9047.

BLOCH, H. \& SEGAL, W. (1955). Viability and multiplication of vaccines in immunization against tuberculosis. American Review of Tuberculosis and Pulmonary Diseases 71, 228-248.

Borremans, M., De Wit, L., Volckaert, G., Ooms, J., De Bruyn, J., Huygen, K., Van Vooren, J. P., Stelandre, M., Verhofstadt, R. \& CONTENT, J. (1989). Cloning, sequence determination, and expression of a 32-kilodalton-protein gene of Mycobacterium tuberculosis. Infection and Immunity 57, 3123-3130.

Closs, O., Harboe, M., Axelsen, N. H., Bunch-Christensen, K. \& MAGNUSSON, M. (1980). The antigens of Mycobacterium bovis, strain BCG, studied by crossed immunoelectrophoresis: a reference system. Scandinavian Journal of Immunology 12, 249-263.

Daniel, T. M. \& ANDerson, P. A. (1978). The isolation by immunoabsorbent affinity chromatography and physicochemical characterization of Mycobacterium tuberculosis antigen 5. American Review of Respiratory Diseases 117, 533-539. 
De Bruyn, J., Bosmans, R., Turneer, M., Weckx, M., Nyabenda, J., VAN Vooren, J. P., Falmagne, P., Wiker, H. G. \& Harboe, M. (1987a). Purification, partial characterization, and identification of a skin-reactive protein antigen of Mycobacterium bovis BCG. Infection and Immunity 55, 245-252.

De Bruyn, J., Huygen, K., Bosmans, R., Fauville, M., Lippens, R., Van Vooren, J. P., Falmagne, P., Weckx, M., Wiker, H. G. Harboe, M. \& Turneer, M. (1987b). Purification, partial characterization and identification of a 32-kDa protein antigen of Mycobacterium bovis BCG. Microbial Pathogenesis 2, 351-366.

De Bruyn, J., Bosmans, R., Nyabenda, J. \& Van Vooren, J. P. (1989). Effect of zinc deficiency on the appearance of two immunodominant protein antigens $(32 \mathrm{kDa}$ and $65 \mathrm{kDa})$ in culture filtrates of mycobacteria. Journal of General Microbiology 135, 79-84.

FukUi, Y., HiRaI, T., UChida, T. \& Yoneda, M. (1965). Extracellular proteins of tubercle bacilli. IV. Alpha and beta antigens as major extracellular protein products and as cellular components of a strain (H37Rv) of Mycobacterium tuberculosis. Biken Journal 8, 189-199.

Garsia, R. J., Hellovist, L., Booth, R. J., RadFord, A. J., Britton, W. J., Astbury, L., Trent, R. J. \& Basten, A. (1989). Homology of the 70-kilodalton antigens from Mycobacterium leprae and Mycobacterium bovis with the Mycobacterium tuberculosis 71-kilodalton antigen and with the conserved heat shock protein 70 of eucaryotes. Infection and Immunity 57, 204-212.

HARBOE, M. \& NAGAI, S. (1984). MPB70, a unique antigen of Mycobacterium bovis BCG. American Review of Respiratory Diseases 129, 444-452.

Harboe, M., Closs, O. \& Deverill, J. (1976). Production of monospecific antisera against antigenic components of Mycobacterium bovis (BCG). Scandinavian Journal of Immunology 5, 861-866.

harboe, M., Closs, O., Bjorvatn, B., Kronvall, G. \& Axelsen, N. H. (1977). Antibody response in rabbits to immunization with Mycobacterium leprae. Infection and Immunity 18, 792-805.

Harboe, M., Coates, A. R. M. \& Hewitt, J. (1985). Characterization of the specificity of monoclonal antibodies against Mycobacterium tuberculosis by crossed immunoelectrophoresis. Scandinavian Journal of Immunology 22, 93-98.

Harboe, M., Nagai, S., Patarroyo, M. E., Torres, M. L., Ramirez, C. \& CRUZ, N. (1986). Properties of proteins MPB64, MPB70, and MPB80 of Mycobacterium bovis BCG. Infection and Immunity 52, 293-302.

Harboe, M., Wiker, H. G., Duncan, R., Garcta, M. M., Dukes, T. W., Brooks, B. W., Turcotte, C. \& NaGaI, S. (1990). Protein Gbased enzyme-linked immunosorbent assay for anti-MPB70 antibodies in bovine tuberculosis. Journal of Clinical Microbiology 28, 913-921.

LAEMmLI, U. K. (1970). Cleavage of structural proteins during the assembly of the head of bacteriophage T4. Nature, London 227, 680685 .

LounatmaA, K. \& BRANDer, E. (1990). Immunoelectron microscopic localization of $22 \mathrm{kDa}$ protein antigen in the surface layer of Mycobacterium bovis BCG strains. In Proceedings of the International Congress for Electron Microscopy, XII, pp. 894-895. San Francisco: San Francisco Press.

Matsuo, K., Yamaguchi, R., Yamazaki, A., Tasaka, H. \& Yamada, T. (1988). Cloning and expression of the Mycobacterium bovis BCG gene for extracellular alpha-antigen. Journal of Bacteriology 170 3847-3854.

Minden, P., Kelleher, P. J., Freed, J. H., Nielsen, L. D., Brennan, P. J., MCPheron, L. \& MCClatchy, J. K. (1984). Immunological evaluation of a component isolated from Mycobacterium bovis BCG with a monoclonal antibody to $M$. bovis BCG. Infection and Immunity 46, $519-525$

Nagai, S., Matsumoto, J. \& Nagasuga, T. (1981). Specific skinreactive protein from culture filtrate of Mycobacterium bovis BCG. Infection and Immunity 31, 1152-1160.
Nagai, S., Wiker, H. G., Harboe, M. \& Kinomoto, M. (1991). Isolation and partial characterization of main protein antigens in the culture fluid of Mycobacterium tuberculosis. Infection and Immunity (in the Press).

Schou, C., Yuan, Z. L., Andersen, A. B. \& Bennedsen, J. (1985). Production and partial characterization of monoclonal hybridoma antibodies to Mycobacterium tuberculosis. Acta Pathologica, Microbiologica et Immunologica Scandinavica, Section C 93, 265-272.

ShinNick, T. M., Vodkin, M. H. \& Williams, J. C. (1988). The Mycobacterium tuberculosis 65-kilodalton antigen is a heat shock protein which corresponds to common antigen and to the Escherichia coli groEL protein. Infection and Immunity 56, 446-451.

TAI, P. C. (1986). Biochemical studies of bacterial protein export. Current Topics in Microbiology and Immunology 125, 43-58.

Tasaka, H., Kiyatani, K. \& Matsuo, Y. (1983). Purification and antigenic specificity of alpha protein (Yoneda and Fukui) from Mycobacterium tuberculosis and Mycobacterium intracellulare. Hiroshima Journal of Medical Sciences 32, 1-8.

Terasaka, K., Yamaguchi, R., Matsuo, K., Yamazaki, A., Nagai, S. \& Yamada, T. (1989). Complete nucleotide sequence of immunogenic protein MPB70 from Mycobacterium bovis BCG. FEMS Microbiology Letters 58, 273-276.

Thole, J. E. R., Dauwerse, H. G., Das, P. K., Groothuis, D. G., Schouls, L. M. \& VAN Embden, J. D. A. (1985). Cloning of Mycobacterium bovis BCG DNA and expression of antigens in Escherichia coli. Infection and Immunity 50, 800-806.

Wiker, H. G., Harboe, M. \& Lea, T. (1986a). Purification and characterization of two protein antigens from the heterogeneous BCG85 complex in Mycobacterium bovis BCG. International Archives of Allergy and Applied Immunology 81, 298-306.

Wiker, H. G., Harboe, M., Nagai, S., Patarroyo, M. E., Ramirez, C. \& CRUZ, N. (1986b). MPB59, a widely cross-reacting protein of Mycobacterium bovis BCG. International Archives of Allergy and Applied Immunology 81, 307-314.

Wiker, H. G., Harboe, M., Bennedsen, J. \& Closs, O. (1988). The antigens of Mycobacterium tuberculosis, H37Rv, studied by crossed immunoelectrophoresis. Comparison with a reference system for Mycobacterium bovis, BCG. Scandinavian Journal of Immunology 27, 223-239.

Wiker, H. G., Harboe, M., Nagai, S. \& Bennedsen, J. (1990a). Quantitative and qualitative studies of the major extra-cellular antigen of Mycobacterium tuberculosis $\mathrm{H} 37 \mathrm{Rv}$ and Mycobacterium bovis BCG. American Review of Respiratory Diseases 141, 830-838.

Wiker, H. G., Sletten, K., Nagai, S. \& Harboe, M. (1990b). Evidence for three separate genes encoding the proteins of the mycobacterial antigen 85 complex. Infection and Immunity 58, 272274.

Yamaguchi, R., Matsuo, K., Yamazaki, A., Nagai, S., Terasaka, K. \& YAMADA, T. (1988). Immunogenic protein MPB57 from Mycobacterium bovis BCG: molecular cloning, nucleotide sequence and expression. FEBS Letters 240, 115-117.

Yamaguchi, R., Matsuo, K., Yamazaki, A., Abe, C., Nagal, S., Terasaka, K. \& Yamada, T. (1989). Cloning and characterization of the gene for immunogenic protein MPB64 of Mycobacterium bovis BCG. Infection and Immunity 57, 283-288.

Young, D. B., IvanYI, J., Cox, J. H. \& Lamb, J. R. (1987). The $65 \mathrm{kDa}$ antigen of mycobacteria - a common bacterial protein? Immunology Today 8, 215-229.

Young, D., Garbe, T., Latigra, L. \& Abou-Zeid, C. (1990). Protein antigens: structure, function and regulation. In Molecular Biology of the Mycobacteria, pp. 1-35. Edited by J. J. McFadden. Surrey University Press. 